Sección Control / Control

Artículos de investigación / Research paper

\title{
Prodiplosis longifila (Diptera: Cecidomyiidae), evolución como plaga y un método para evaluar sus poblaciones en tomate
}

\author{
Prodiplosis longifila (Diptera: Cecidomyiidae) evolution as pest and a method to assess \\ its populations in tomato
}

\author{
(iD) FRANCIS GERAUD-POUEY ${ }^{1 *}$, iD AlBERTO GARCES ${ }^{2}$, \\ iDNANCY CONTRERAS ${ }^{2}$, iDJUAN E. GERAUD-CHIRINOS ${ }^{1}$
}

${ }^{1}$ Universidad del Zulia. Maracaibo, República Bolivariana de Venezuela. fgeraudp@gmail.com,jgeraudc@gmail.com

${ }^{2}$ Universidad Agraria del Ecuador, Milagro. Ecuadoragarces@uagraria.edu.ec,nancycontrerasm2@hotmail.com

\begin{abstract}
Autor de correspondencia
Francis Geraud-Pouey. Universidad del Zulia. Maracaibo 4001, República Bolivariana de Venezuela. fgeraudp@gmail.com*

Citación sugerida

GERAUD-POUEY, F. GARCES, A.; CONTRERAS, N.; GERAUD-CHIRINOS, J. E. 2022. Prodiplosis longifila (Diptera: Cecidomyiidae), evolución como plaga y un método para evaluar sus poblaciones en tomate. Revista Colombiana de Entomología 48 (1): e 7807. https:// doi.org/10.25100/socolen.v48i1.7807

Recibido: 22-Sep-2021

Aceptado: 28-Oct-2021

Publicado: 28-Ene-2022

Revista Colombiana de Entomología ISSN (Print): 0120-0488

ISSN (On Line): 2665-4385

https://revistacolombianaentomologia.univalle.edu.co

Open access

(c) (i) (2)(2) BY-NC-SA 4.0
\end{abstract}

Publishers: Sociedad Colombiana de Entomología SOCOLEN (Bogotá, D. C., Colombia) https://www.socolen.org.co

Universidad del Valle (Cali, Colombia)

https://www.univalle.edu.co

(C) 2021 Sociedad Colombiana de Entomología SOCOLEN y Universidad del Valle - Univalle
Resumen: Desde mediados de la década de 1980, Prodiplosis longifila se ha convertido en el principal problema entomológico del tomate en Ecuador. El escaso conocimiento bioecológico, para racionalizar su manejo, ha llevado al excesivo uso de plaguicidas. Siendo tanto el insecto como el tomate de origen neotropical, su reciente evolución como problema fitosanitario plantea interrogantes que ameritan revisar la historia del caso. Estudiar sus poblaciones y los factores bióticos que las regulan, es fundamental para responderlas. Una aproximación metodológica consiste en estimar el porcentaje de plantas afectadas, complementado con el número de insectos en muestras periódicas de follaje infestado, durante el ciclo del cultivo. La manera tradicional de llevar las muestras al laboratorio envueltas en papel toalla, dentro de bolsas de polietileno, resultó ineficiente al quedar muchas larvas atrapadas en la humedad del interior de las bolsas o tejer capullo sobre el papel, complicando su recuperación para conteo y cría hasta emergencia de adultos y parasitoides. Así, aprovechando el hábito de pupar en el suelo, se diseñó un contenedor de muestras con suelo cernido en el fondo, donde las larvas que han completado su desarrollo se dejan caer para tejer sus capullos, facilitando su recuperación mediante cernido. La economía de tiempo en los procesamientos de muestras ( 5 veces $<$ tiempo), los números de individuos obtenidos de las muestras, así como adultos y parasitoides emergido de sus crías $(5,0-7,7$ veces $>$ número), complementado con coeficientes de correlación de recuperados vs. adultos y parasitoides criados (1,2-1,9 veces $>$ ) para los contenedores con suelo comparado con bolsas de polietileno, indican la ventaja de este nuevo método.

Palabras clave: Mosquita de brotes, infestaciones, negrita del tomate, muestreos, poblaciones.

Abstract: Since mid 1980's Prodiplosis longifila became the main entomological problem of tomato crops in Ecuador. The lack of bioecological knowledge to guide its rational management has resulted in excessive use of insecticides. Being both the insect and tomato of neotropical origin, the former's recent evolution as phytosanitary problem raises challenging questions that deserves revising the case history. Population studies including biotic regulatory factors are fundamental to answer them. A methodological approach consists of estimating the percentage of infested plants complemented with the number of insects in periodical samples along the crop phenology. The usual way of carrying field samples to the laboratory wrapped in paper towel inside plastic bags resulted inefficient since many larvae were trapped in the humidity inside the bags or spun their cocoons on the paper towel, making their retrieval for rearing and obtaining adults and parasitoids, time consuming. Thus, taking advantage of the insect's habit to pupate in the soil, a sample container with sieved soil in the bottom was designed so larvae that completed development drop to the soil to spin their cocoons, which are easily recovered by sieving. The time economy $(5 \mathrm{x}<$ time $)$, the number of individuals obtained from the samples, as well as adults reared from them $(5.0-7.7 \mathrm{x}>$ number), complemented with correlation coefficients of obtained vs. adults and parasitoids, reared from the obtained $(1.2-1.9 \mathrm{x}>$ ) for containers with soil compared with plastic bags, shows the advantages of this new sampling method.

Keywords: Tomato bud midge, infestations, populations, sampling. 


\section{Introducción}

Desde hace algo más de tres décadas, "la negrita", "liendrilla” o “tostón”, Prodiplosis longifila Gagné, 1986 (Diptera: Cecidomyiidae) es considerada como el principal problema entomológico del tomate, Solanum lycopersicum L., 1753, en Ecuador (Valarezo et al. 2003). Los primeros brotes poblacionales con "fuertes" infestaciones por este insecto habían sido detectados en septiembre de 1979 en Chilca, departamento de Lima, Perú, dañando frutos de un cultivo de tomate cercano a campos de alfalfa (Medicago sativa L., 1753) y papa (Solanum tuberosum L., 1753), los cuales tenían más del 60\% de brotes con larvas de este insecto (Díaz 1981). En los siguientes dos años se registraron brotes poblacionales en Pampilla-Cañete, con infestaciones del 90\% de los frutos y en la zona hortícola de San Felipe (ambas localidades del departamento de Lima), así como en Guadalupe (departamento de La Libertad), ante cuyos altos niveles de infestación en tomate, los agricultores se vieron impelidos a realizar aplicaciones semanales de insecticidas a dosis más elevadas que las recomendadas (Díaz 1981).

En Ecuador, P. longifila fue detectada en 1986 en el cantón Arenillas (provincia de El Oro), y seguidamente su presencia determinada en otras 11 provincias, principalmente en la zona costera y valles interandinos, con una distribución altitudinal desde nivel del mar hasta $1800 \mathrm{msnm}$ (Valarezo et al. 2003). Siendo Arenillas limítrofe con Perú, donde primero se le mencionó como problema fitosanitario, estos autores supusieron que esa era la "ruta de entrada" del insecto. Al igual que durante los inicios del problema en Perú (Díaz 1981), la totalidad de los productores aplicaron indiscriminadamente productos químicos, con todos los riesgos de secuelas ambientales y en salud humana, llegando inclusive al abandono del cultivo de tomate en algunas zonas, debido a la pérdida de rentabilidad (Valarezo et al. 2003). Aunque en Ecuador también se le conocen varias especies de plantas hospederas cultivadas, solo ha sido limitante en la producción de tomate (Valarezo et al. 2003), situación que no parece haber cambiado (Chirinos . 2020). En Colombia, los primeros daños por P. longifila en tomate se observaron en 1987 (Espinosa y Toro 2015). Pero tardó alrededor de una década en convertirse en una de las plagas más limitantes en las zonas tomateras de Valle del Cauca, cuyo manejo se basó en el uso indiscriminado de plaguicidas químicos [Delgado (1998) citado por Mena et al. (2014)]; tal como ocurrió en Perú y Ecuador. Hasta donde se conoce, $P$. longifila no se ha encontrado en Venezuela, aunque en los últimos 30 años se han conducido inventario entomofaunístico en tomate (Geraud-Pouey et al. 1995), de begomovirus en ese mismo cultivo (Geraud-Pouey et al. 2015) y de moscas blancas del complejo Bemisia tabaci (Gennadius, 1889) en tomate y otras hortalizas (Romay et al. 2016), cubriendo en cada caso gran parte del territorio nacional.

Recientemente, $P$. longifila ha sido incluida en una lista de organismos dañinos al tomate, con riesgos de ser introducidos a Europa, mediante importación de frutos (Grousset et al. 2015), lo cual podría convertirse en alerta fitosanitaria, potencialmente restrictiva de importaciones de ese y otros productos agrícolas.

Un aspecto importante que plantean estos abruptos cambios de estatus de una especie como plaga, por acentuados y atípicos aumentos poblacionales, tiene que ver con los factores que pueden haberlos producido. Tanto $P$. longifila (Gagné 1986) como el tomate (Peralta y Spooner 2000), son de ori- gen neotropical y han coexistido en la vertiente de los Andes hacia el Pacífico, donde se ha cultivado el tomate con cierta intensidad por al menos un siglo. Esto impone la necesidad de revisar los cambios ocurridos en los manejos agronómicos y en especial con el uso de insecticidas a partir de la entrada al mercado de los organosintéticos (1947), los cuales, desde muy temprano causaron serios problemas en el manejo de plagas en esa región, debido a su uso "indiscriminado" (Herrera 2010). La continuidad temporal entre los eventos agronómicos anteriores y el inicio de los problemas causados por $P$. longifila, sugieren que este fuese un caso de "plaga secundaria o potencial" elevada de estatus debido a desbalances en el control por sus enemigos naturales, a consecuencia de dicho uso de insecticidas para controlar otras especies consideradas como "plagas". Esto coincide con la antes mencionada intensificación de aplicaciones de insecticidas para controlar los brotes de esta nueva "plaga" (Díaz 1981), lo cual implica que trataron de controlar el problema con el mismo método con el que probablemente lo causaron.

Todo esto enlaza con el problema en que ha devenido el uso actual de insecticidas en tomate, lo cual ha sido documentado recientemente para este y otros cultivos en el sur del Ecuador (Chirinos et al. 2020). Tal como refieren los autores, si bien los agricultores entrevistados, mencionaron otros insectos como problema en el cultivo del tomate, tales como moscas blancas (Hemiptera: Aleyrodidae) y Liriomyza spp. (Diptera: Agromyzidae), P. longifila es la principal razón por la cual aplican insecticidas, generalmente a partir de pocos días después del trasplante, alcanzando en promedio 2,8 aplicaciones/semana (amplitud 2-4). Aunque con algunas diferencias de especies objetivos de aplicaciones de insecticidas, similares situaciones de intensidad de uso de estos agroquímicos han sido documentadas para tomate y otros cultivos en Venezuela (Chirinos y Geraud-Pouey 2011). Para el caso de $P$. longifila, comparado con la cantidad de insecticidas aplicados, existe poca información científica acerca de los aspectos bioecológicos que sirvan de base para orientar el manejo más racional de este importante problema fitosanitario. Una parte apreciable de la información disponible en la red corresponde a publicaciones divulgativas y boletines técnicos de empresas representantes de agroquímicos, generalmente recomendando algunos de sus productos para controlar al insecto considerado plaga.

Lo anterior plantea la posible alteración del control biológico natural por uso inadecuado de plaguicidas, fenómeno documentado para otros casos, incluyendo evidencias experimentales (Doutt y Smith 1971; Oatman y Kennedy 1976; Chirinos y Geraud-Pouey 1996; Chirinos et al. 2014). En el caso de $P$. longifila, ha sido referida la existencia de parasitoides del género Synopeas (Hymenoptera: Platygastridae), pero con efectos variables debido a la interferencia de acuerdo con la intensidad de uso de plaguicidas (Peña et al. 1990; Valarezo et al. 2003; Díaz-Silva 2011; Hernández 2014; Hernández et al. 2015). Ante la magnitud y complejidad de este problema fitosanitario, se le está prestando atención a la genética de poblaciones de este insecto, para intentar aclarar si se trata de una especie con amplitud de plantas hospederas o un complejo de especies crípticas o biotipos cada uno particularmente relacionado con ciertas especies de plantas hospederas (Espinosa y Toro 2012; Ortega et al. 2014). En búsqueda de alternativas adicionales de manejo, también se ha considerado la resistencia de plantas hospederas (Mena et al. 2014), al igual que la aplicación de entomopatógenos como bioinsecticidas (Ce- 
dano y Cubas 2012), hasta ahora con efectos muy limitados.

A P. longifila se le conoce una amplia distribución geográfica neotropical alcanzando el sur de Florida, EE.UU., al igual que diversidad de plantas hospederas en diferentes familias botánicas (Díaz 1981; Gagné 1986; Valarezo et al. 2003; Díaz-Silva 2011; Hernández 2014; Hernández et al. 2015). Así, lo que Rainwater (1934) mencionó en Florida como Contarinia gossypii Felt, 1908, dañando brotes de algodón silvestre, Gossypium sp., podría tratarse de $P$. longifila, ya que "C. gossypii puede no ocurrir en Florida"; mientras que el material tipo para la descripción de $P$. longifila proviene de algodón silvestre del condado de Monroe al Sur de Florida, EE. UU. (Gagné 1986). Posteriormente, en esa misma región, $P$. longifila causó daños en flores de lima Tahití, Citrus latifolia (Tanaka, 1976) (Peña et al. 1987, Peña et al. 1989; Peña y Duncan 1992). Actualmente no se le considera de importancia como problema fitosanitario, en buena parte debido a la efectividad del control biológico natural (Peña et al. 1990).

La manera cómo evolucionaron los problemas entomológicos causados por $P$. longifila en Perú, presentan una interesante coincidencia cronológica con el caso de Florida. Las primeras referencias se le atribuyen a Wille (1930, 1952; citado por Díaz-Silva 2011) quién supuestamente la mencionó como mosca Cecidomyiidae del género Contarinia Róndani, 1860, dañando terminales y flores de alfalfa. Es probable que la primera de esas referencias se trate de un informe de Wille como entomólogo Jefe la Estación Experimental Agrícola de la Molina con fecha "1930b" y la segunda, es una breve mención en su libro "Entomología Agrícola del Perú", ambos referidos y listados por Lamas (1982). En la colección entomológica de esa misma Estación Experimental, se preservaron especímenes de Cecidomyiidae colectados entre 1958 y 1975 en brotes de higuerilla, Ricinus communis L., 1753, pallar, Phaseolus lunatus L., 1753, papa y alfalfa, identificados en su mayoría por R.J. Gagné, como diferentes especies del género Contarinia, aclarándose posteriormente que esos especímenes corresponden a una nueva especie del género Prodiplosis Felt, 1908 (Díaz 1981), la cual es la primera mención de esa especie de Prodiplosis en Perú. Esos especímenes fueron luego revisados por el mismo Gagné coincidiendo con lo que describió como P. longifila (Gagné 1986). Aparentemente ninguno de esos registros estuvo asociado con importantes infestaciones.

Problemas entomológicos con esta relevancia, generados por elevación de especies autóctonas a niveles de plagas, demandan en primera instancia revisar la historia de dichos cambios, si se consideran los manejos agronómicos dentro de los cuales ocurrieron, parte de lo cual aquí se documenta. Seguidamente, se deben realizar estudios de poblaciones y de factores especialmente bióticos que las regulan, para tratar de comprender las causas de esos cambios, de modo de orientar la búsqueda de manejos más racionales desde los puntos de vista ecológico y socioeconómico. Los estudios de poblaciones requieren de metodologías que permitan evaluarlas con eficiencia e idoneidad. Si bien existen experiencias en evaluar poblaciones y daños causados por este insecto en diferentes cultivos tales como cítricos (Peña et al. 1987; Peña y Duncan 1992), espárragos (Díaz-Silva 2011) y tomate (Valarezo et al. 2003), algunos de los métodos utilizan la captura en trampas pegantes, de adultos tanto de $P$. longifila como de sus parasitoides que emergen del suelo en los campos de cultivo. El manejo en campo y laboratorio de este tipo de trampa tiende a ser engorroso, además que limita la identificación y recuperación de los insectos atrapados en el pegamento, especialmente los de tamaño tan pequeño, restringiendo la documentación de los aspectos taxonómicos y de biodiversidad involucrados en este tipo de estudios.

El objetivo del presente trabajo fue documentar la evolución histórica de $P$. longifila como problema fitosanitario e iniciar estudios de sus poblaciones y factores bióticos reguladores de las mismas en cultivos de tomate. No obstante, las deformaciones que el insecto le causa al follaje tierno dificultan considerablemente su revisión en el campo, así como de las muestras traídas al laboratorio para los conteos directos sobre dichas muestras. Esto junto con las complicaciones que plantea transportarlas al laboratorio dentro de bolsas de polietileno, detectadas al inicio de esta investigación, indujo a evaluar una alternativa metodológica en busca de mayor eficiencia en esas evaluaciones.

\section{Materiales y métodos}

A la par de revisar la evolución del caso de $P$. longifila durante los últimos 50 años, se condujo una investigación de campo y laboratorio, acerca de fluctuaciones poblacionales y relación con la planta de tomate, durante el período junio 2015-enero 2016 (época de sequía hasta entrada de lluvias con esporádicas precipitaciones). Los trabajos de campo se realizaron en el Campo Experimental El Misionero de la Universidad Agraria del Ecuador, Sede Milagro, cantón Milagro, provincia de Guayas, República del Ecuador $\left(02^{\circ} 08^{\prime} 45^{\prime \prime} \mathrm{S} ; 7^{\circ} 34^{\prime} 21^{\prime \prime O}\right)$. El procesamiento de las muestras de campo se hizo en el Laboratorio de Entomología $\left(25-26^{\circ} \mathrm{C}, \mathrm{HR} 80 \%\right.$, iluminación natural complementada con luz artificial durante horas diurnas) del Campus Guayaquil.

Para los fines de esta investigación, se sembraron dos parcelas de tomate cv. Floradade (Agrosad, Quito, Ecuador), preparadas en camas (camellones) de 0,7 $\mathrm{m}$ de ancho, separadas por surcos de $0,3 \mathrm{~m}$ de ancho. La primera parcela de aproximadamente $700 \mathrm{~m}^{2}(28 \times 25 \mathrm{~m})$, dividida en 20 subparcelas (unidades de observación y muestreo) con 5 camas de $7 \mathrm{~m}$ de largo, trasplantada el 17 julio 2015 y la segunda de unos $750 \mathrm{~m}^{2}(30 \times 25 \mathrm{~m})$, dividida en 15 subparcelas con igual número de camas, pero de $10 \mathrm{~m}$ de largo, trasplantada el 10 noviembre 2015. Ambas parcelas fueron fertirregadas mediante mangueras de goteo, una a lo largo del centro de cada cama. Los trasplantes se alternaron en dos hileras, uno a cada lado de la manguera de riego por goteo, con distancias de $0,4 \mathrm{~m}$ entre puntos de trasplante (tresbolillo). Otros manejos agronómicos se hicieron a la usanza de la zona, pero sin controlar insectos. La fertilización se hizo a través del riego, dos veces por semana con $0,18 \mathrm{~kg} /$ ha de Nitrógeno, $0,52 \mathrm{~kg} /$ ha de Ácido Fosfórico, 0,18 kg/ha de Potasio y $0,02 \mathrm{~kg} / \mathrm{ha}$ de Sulfato de Magnesio. La competencia por plantas arvenses fue manejada mediante deshierbe manual.

Las evaluaciones consistieron de observaciones en campo del porcentaje de plantas con brotes y/o folíolos con daños recientes por $P$. longifila, independientemente de la intensidad del daño, de las cuales se separaba un número variable de ellos de acuerdo a la disponibilidad para llevar al laboratorio, con el fin de realizar conteos de individuos emergidos de las mismas, discriminándolos taxonómicamente y estableciendo sus funciones tróficas (posiciones en cadenas de consumo), basado en lo cual seguir las fluctuaciones poblacionales del fitófago y sus parasitoides.

En muestreos preliminares, las muestras se colocaron envueltas con papel toalla dentro de bolsas de polietileno, méto- 
do comúnmente utilizado para traslado al laboratorio dentro de una hielera atemperada (aproximadamente $18{ }^{\circ} \mathrm{C}$ ). Allí se observó que parte de las larvas de $P$. longifila que habían completado su desarrollo (tercer estadío), al abandonar el follaje buscando ir al suelo a pupar (Peña et al. 1989), quedaban atrapadas en la humedad del interior de la bolsa, y otras tejían sus capullos entre las fibras del papel toalla, ambas haciendo muy laborioso e ineficiente su recuperación para conteo y cría hasta emergencia de adultos de $P$. longifila y parasitoides.

Como alternativa, aprovechando el hábito del insecto de dejarse caer para pupar en el suelo, se ideó un contenedor para muestras, que facilitara la recuperación de los individuos que abandonan el follaje muestreado. Dicho contenedor consiste en un envase de plástico rígido $(10 \times 10 \times 6 \mathrm{~cm}$ largo $\mathrm{x}$ ancho $\mathrm{x}$ alto, medidas externas, $400 \mathrm{cc}$ de capacidad) con tapa a presión, dentro del cual se coloca una rejilla mosquitero de aluminio sostenida a presión contra las paredes internas a me- dia altura del envase sobre la cual se colocaba la muestra y el fondo del mismo recubierto por una capa de suelo (3-4 mm de espesor) finamente cernido (tamiz de $500 \mu \mathrm{m}$ ), para que las larvas que salen a pupar dejándose caer, lo hagan dentro del suelo, tal como ocurre en la naturaleza (Fig. 1 E y F).

En la primera parcela, durante las tres primeras semanas se tomaron muestras envueltas en papel toalla dentro de bolsas de polietileno (Fig. 2 A y C), para compararlas con muestras colocadas en contenedores con suelo (Fig. 3 A-C). Los muestreos con contenedores se continuaron hasta el final del trabajo de campo. Las muestras eran trasladas al laboratorio dentro de hieleras atemperadas. En la primera parcela se tomaron 11 muestras-semana, mientras que en la segunda solo se le pudieron tomar cinco muestras-semana, debido a alta mortalidad de plantas a consecuencia de infección por un hongo del género Verticillium (Prof. Winston Fitopatólogo de la UAE, Guayaquil, reporte de diagnóstico).

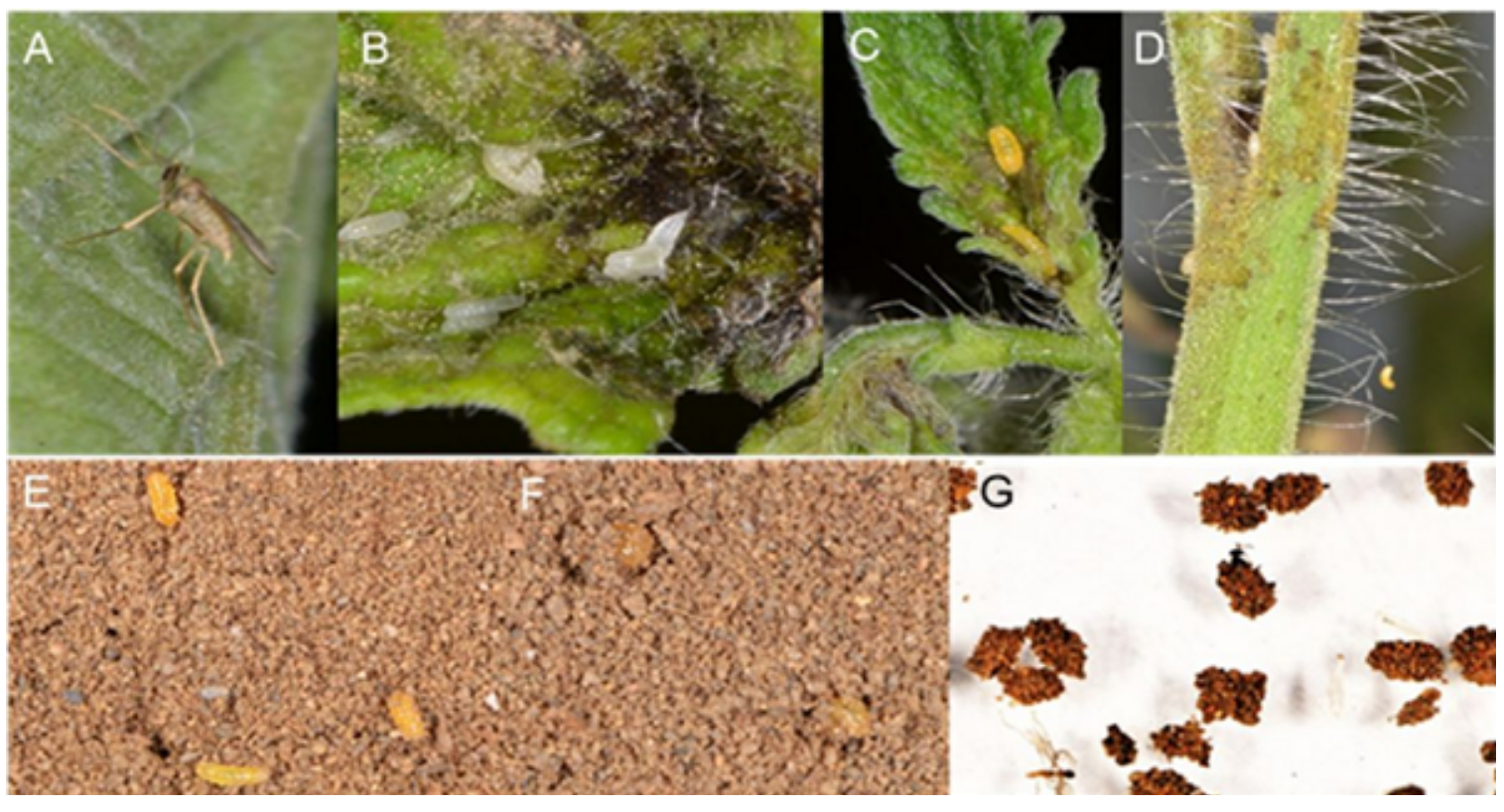

Figura 1. A. Hembra adulta de Prodiplosis longifila. B. Larvas dañando folíolo tierno. C. Larvas de finales del tercer estadío (L3). D. L3 abandonando la planta para ir al suelo a pupar. E. L3 sobre el suelo. F. L3 tejiendo capullo dentro del suelo. G. Capullos recubiertos de partículas de suelo.

En el caso de contenedores, después de pesadas las muestras se retornaron a sus respectivos contenedores, pero sin cerrar completamente la tapa para evitar desarrollo de hongos por exceso de humedad. Las larvas que han completado el tercer estadío se dejan caer al fondo del envase y pupan en el suelo (Fig. 1 E-G). Pasados 4 días después del muestreo, se cernía el suelo de cada contenedor y se recuperaban los puparios retenidos en el tamiz (Fig. 3 D y E). Análogamente al caso de las larvas y puparios recuperados de las bolsas de polietileno, para este otro método de manejo de muestras, los puparios constituyeron los individuos obtenidos de las muestras. Después de contados, se colocaban dentro de viales de vidrio (10 dram) tapados con algodón, hasta la emergencia de las mosquitas (Fig. 3 F-I). Los adultos no alimentados sobreviven por muy corto tiempo, lo cual facilita el conteo y su eliminación de los viales, donde después se retornaban los puparios, en espera de la emergencia de parasitoides, lo cual tardan algunos días adicionales.
En ambos casos, los adultos emergidos de las crías fueron discriminados taxonómicamente y contados. En caso de que se requiera obtener adultos de parasitoides para fines taxonómicos hay que permitir que emerjan de los puparios; pero si ya se les conoce, es posible adelantar el procedimiento, disolviendo con hipoclorito de sodio (5\%) la seda de los capullos que conforman los puparios, lo que libera las pupas de los parasitoides allí contenidos, permitiendo su identificación y conteo. Dada la variación de los pesos de las muestras, los números de individuos obtenidos de cada muestra fueron uniformizados para 10 gramos de peso fresco (No. uniformizado $=$ No. obtenidos x $10 \mathrm{~g} /$ peso fresco).

Observaciones acerca de la biología de $P$. longifila, descripción de daños causados a la planta de tomate y evolución de las infestaciones en campo. Con la finalidad de establecer las partes de la planta de tomate que aportan información acerca de la población actual al momento de cada 
muestreo, se realizaron observaciones de campo y laboratorio relacionadas con la biología de $P$. longifila y sus parasitoides, así como de las infestaciones y daños causados a la planta siguiendo su fenología en las parcelas bajo estudio. Los aspectos biológicos fueron cubiertos mediante observaciones de brotes dañados en campo utilizando lupas de bolsillo (10x y 20x), complementado con observaciones bajo microscopio estereoscópico en el laboratorio (6,5-40x). Basado en previas investigaciones (Peña et al. 1989; Díaz-Silva 2011; Hernández 2014), se diferenciaron estadíos de desarrollo mediante tamaño y coloración de las larvas. La evolución del desarrollo se observó durante el procesamiento de las muestras en el laboratorio. Las infestaciones y daños se documentaron mediante registro de porcentajes de plantas con daños, complementado con fotografías de los daños.

Identificación de $\boldsymbol{P}$. longifila y sus parasitoides. Adultos de $P$. longifila y parasitoides obtenidos de las muestras de campo procesadas en el laboratorio, preservadas en alcohol etílico al $96 \%$, se separaron por morfotipos. La identificación de $P$. longifila se hizo siguiendo la clave y descripción proporcionadas por Gagné (1986). Para los parasitoides preservados de la misma manera, se utilizaron como referencias las descripciones provistas por Hernández (2014). La identificación de los parasitoides de la familia Platygastridae (Hymenoptera) fue complementada por Peter Neerup Buhl (Natural History Museum of Denmark, Copenhaguen, Dinamarca). Para la identificación de $P$. longifila, la separación e identificación de algunos de los morfotipos de parasitoides, así como fotografiado de los mismos, se utilizó un microscopio estereoscópico $(10-80 x)$ de alta resolución con cámara fotográfica digital. Muestras en alcohol de los especímenes identificados están depositadas en la Colección Entomológica de la UAE, en Guayaquil.
Comparación de los manejos de muestras. Para comparar los dos manejos de muestras (bolsas de polietileno con muestra envuelta en papel toalla vs. contenedores de muestras con suelo), se estimó el tiempo de toma en campo, la separación y conteo de los insectos en el laboratorio, para cada uno de ellos. Además, para evaluar rendimiento en número de insectos según el manejo de muestras, se cuantificó: 1) individuos de $P$. longifila obtenidos vivos después de emerger del follaje muestreado y puestos en cría; 2) adultos de la misma especie emergidos de las crías; 3) adultos de parasitoides (discriminados por familia) emergidos de las mismas crías; 4) adultos totales ( . longifila + parasitoides); 5) tasa de adultos totales emergidos de los puparios (= numeral 4/numeral 1); $6)$ porcentaje de parasitismo con base a adultos totales $[=(\mathrm{nu}-$ meral 3)x100/(numeral 4)]. Con el fin de evaluar el grado de relación de algunas de esas variables, se calculó la correlación de Pearson para: 1) numeral 1 vs. 4 ; 2) numeral 1 vs. 2; 3) numeral 1 vs. 3 ; 4) relación de cada taxón de parasitoide discriminado (Synopeas spp. y Chalcidoidea) vs. parasitismo total, para medir la importancia relativa de cada taxón. Además, mediante regresiones simples para la primera y segunda de estas últimas comparaciones, se evaluaron los ajustes a varios modelos para conocer la mejor interpretación de esas relaciones como medidas complementarias de la eficiencia de los dos métodos de muestreos. Los análisis se hicieron utilizando el programa SPSS de IBM Corporation (2011).

Fluctuación poblacional de $\boldsymbol{P}$. longifila y sus parasitoides. Para visualizar como fluctuó la población de $P$. longifila y su relación con la incidencia de parasitismo total a lo largo de la fenología del cultivo, se calcularon medias y error estándar de medias, para número de puparios recuperados de las muestras en contenedores, así como de adultos de $P$. longifila y parasitoides totales (Synopeas spp. + Chalcidoidea) obteni-

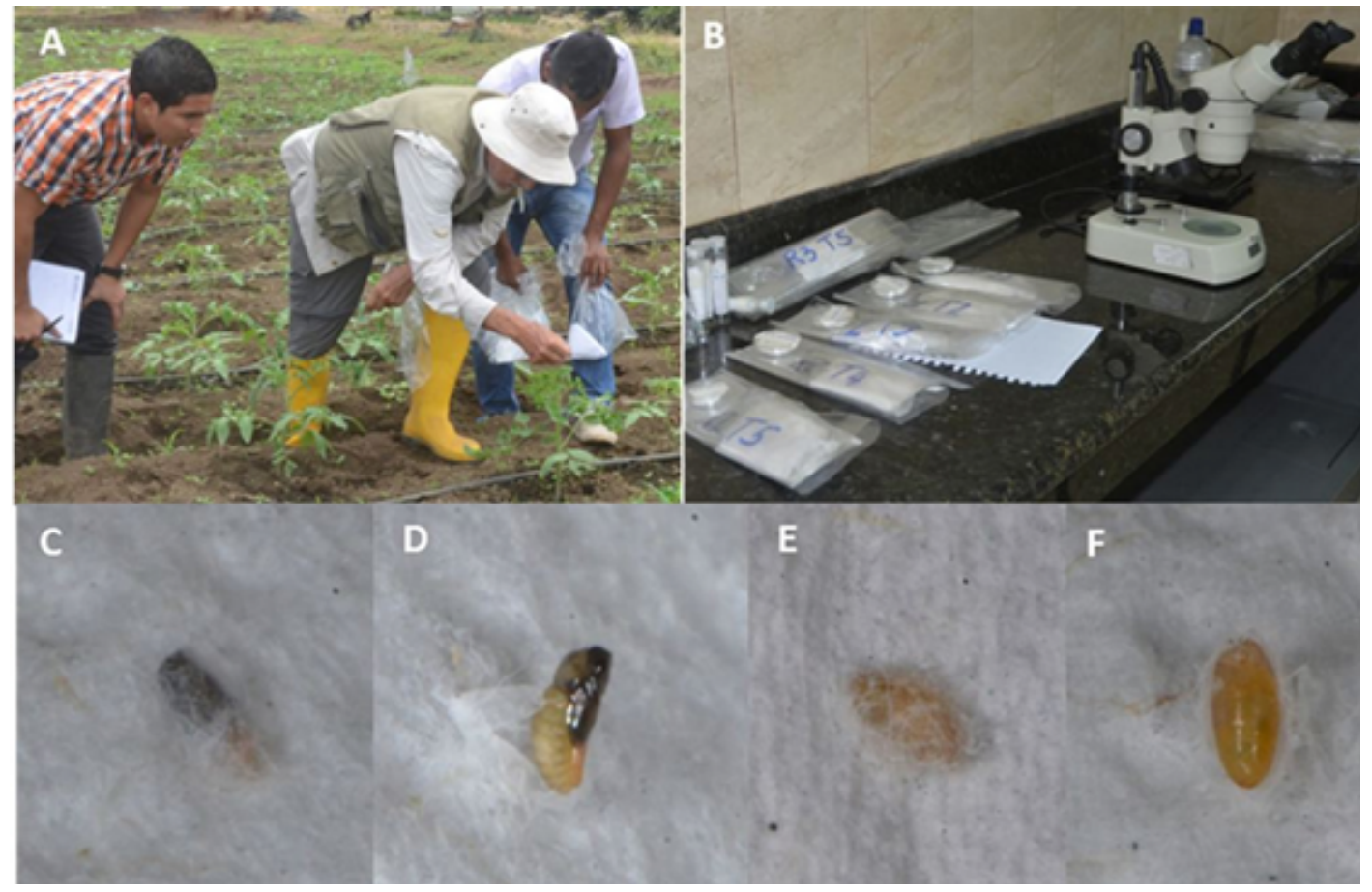

Figura 2. Muestreo de Prodiplosis longifila en tomate, utilizando bolsas de polietileno con papel toalla. A. Toma de muestra. B. Muestras en espera de emergencia de L3 en laboratorio. C. Pupario de P. longifila sobre papel toalla. D. Pupa de P. longifila extraída del pupario. E. Pupario de Synopeas sp. dentro del pupario de P. longifila sobre papel toalla. F. Pupario de Synopeas sp. 
dos de las crías dentro los viales en el laboratorio. Con estos se calcularon los porcentajes de parasitismo [\% Parasitismo = $100 \mathrm{x}$ parasitoides totales /adultos totales]. Relacionar el parasitismo a adultos totales en vez de a puparios de $P$. longifila, evita que otras causas de mortalidad debido a las condiciones de las crías en el laboratorio, enmascaren el efecto del parasitismo. Con esa información se construyeron gráficas de poblaciones para ambas parcelas.
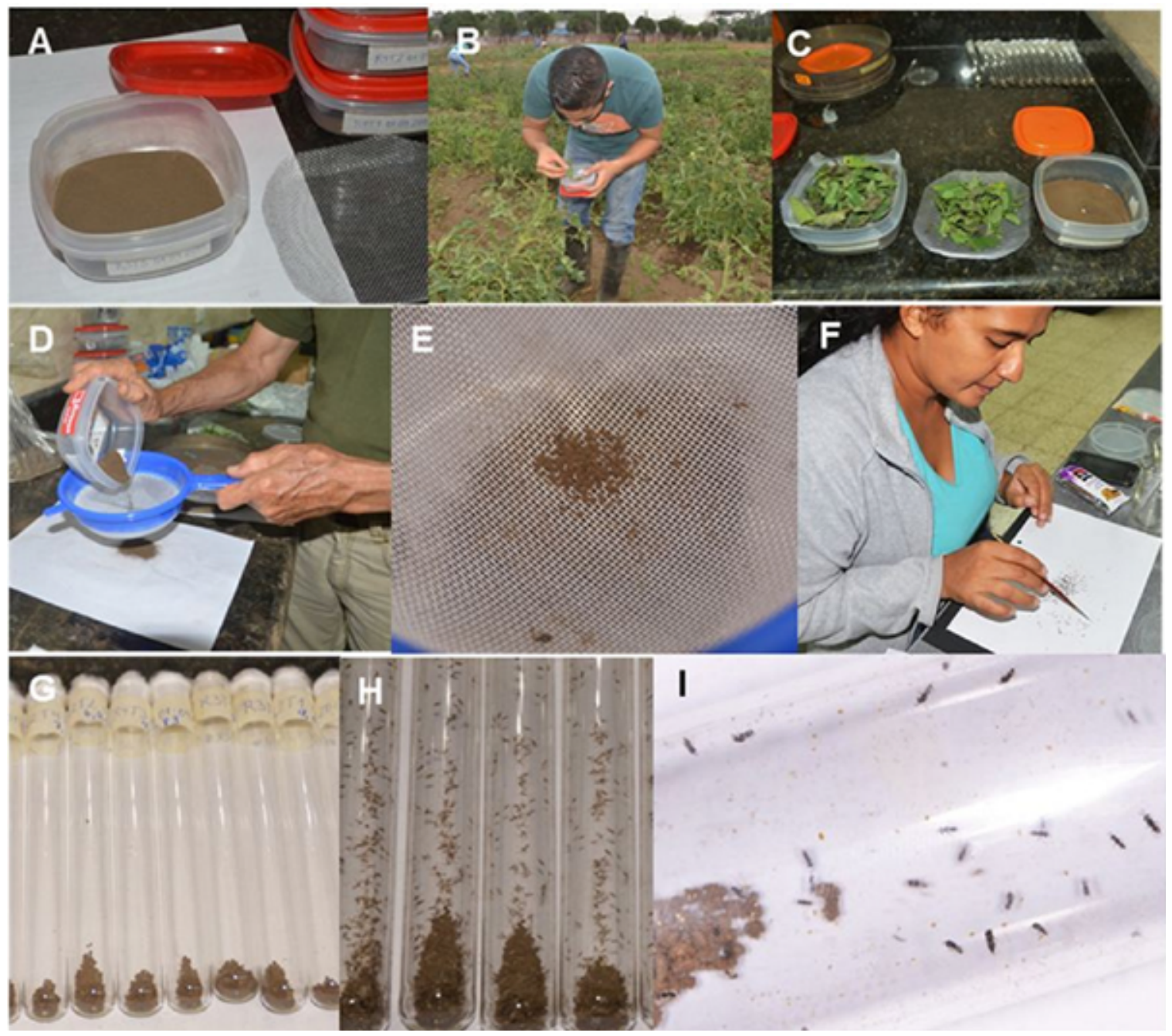

Figura 3. Muestreo de Prodiplosis longifila en tomate, utilizando contenedor de muestra con suelo en el fondo. A. Envase contenedor de muestra con suelo y rejilla mosquitero de aluminio. B. Toma de muestra en campo. C. Muestras listas para pesaje en laboratorio. D. Cernido del suelo de la muestra. E. Puparios de $P$. longifila recubiertos de arena, separados por el cernidor. F. Conteo de puparios obtenidos de la muestra. G. Viales que contienen puparios. H. Viales con adultos de P. longifila emergidos de los puparios. I. Adultos de parasitoides emergidos de los puparios de P. longifila.

\section{Resultados y discusión}

La manera un tanto abrupta como emergieron y evolucionaron los problemas fitosanitarios causados por P. longifila en la zona andina durante los últimos cuatro decenios, plantea interrogantes en cuanto a impacto de manejos agronómicos, especialmente en lo que refiere al uso de plaguicidas, sin descartar la posible diversificación genética de las poblaciones naturales a lo largo de esa amplia distribución geográfica, con sus variantes climáticas y condiciones agronómicas, hasta el punto de quizás conformarse un complejo de especies crípticas (Dhileepan et al. 2017), cuya investigación debe ser acometida para partir de bases más objetivas para su manejo. De principio, se tiende a pensar en la dispersión geográfica del organismo causal del problema fitosanitario en cuestión, a partir de su "zona de origen" (donde primero se le mencionó causando problemas) (Valarezo et al. 2003). No obstante, la elevación de su estatus coincidente con la intensificación del uso de plaguicidas organosintéticos (Díaz 1981; Herrera 2010; Chirinos et al. 2020) apunta hacia el clásico "síndrome de plaguicidas" (Doutt y Smith 1971). El largo tiempo para que este cambio se iniciara en Perú (49 años), desde que aparentemente fue el primer reporte por Wille (Díaz-Silva 2011), junto con la ignorada diversidad de especies de parasitoides posteriormente referidas en Colombia (Hernández 2014), la zona más recientemente afectada, complementado con la aquí informada (ver más adelante), sugieren que este complejo de especies (fitófago-parasitoides) ya existía en esta zona de los Andes, y el fitófago comenzó a ser tomado en cuenta al hacercon el insecto hospedero, logrando especímenes en buenas 
se aparente, a causa de los citados desbalances de su control biológico natural inducidos por plaguicidas.

Observaciones acerca de la biología de $P$. longifila, descripción de daños que causa en tomate y evolución de las infestaciones en campo. El adulto de este insecto es de hábitos crepusculares (Peña et al. 1989). En el laboratorio, comenzaban a emerger de los puparios, entre 4 y 5 pm, 4-7 días después de cernidos de los contenedores de las muestras. Los adultos no alimentados sobrevivieron 1-2 días. En el campo, las hembras (Fig. 1 A) depositan sus huevos en brotes y folíolos muy tiernos aún no desplegados, generalmente ubicándolos hacia la parte basal de los mismos, donde al eclosionar, las larvas se alimentan raspando la superficie del haz, cuyo daño la torna negruzca. Se supone que de allí deriva el nombre común de "la negrita del tomate", uno bajo los cuales se le conoce en Ecuador, (Valarezo et al. 2003) (Fig. 1 B). Al completar su desarrollo, la larva de tercer estadío se torna de color amarillo intenso (Fig. 1 C) y se deja caer de la planta para ir al suelo a pupar (Fig.1 D y E), donde penetra unos pocos milímetros y teje su capullo o pupario recubierto de partículas de suelo (Fig. 1 F y G). En flores de lima Tahití, $C$. latifolia, en el sur de Florida, el desarrollo de huevo a adulto tardó aproximadamente 12-17 días y la longevidad del adulto sin alimentar fue de 1 día, mientras que alimentado con solución de miel de abeja fue de 8-9 días (Peña et al. 1989).
A consecuencia del daño, bajo altas infestaciones, los órganos afectados se deforman de tal manera que con frecuencia las hojas nuevas quedan reducidas al raquis con folíolos deformes y en buena parte retorcidos y necrosados (Fig. 4 A y B). Aunque el daño comienza por los brotes apicales, también dañan las yemas axilares, impidiendo la ramificación de la planta, la axila queda con la superficie necrosada, donde con frecuencia se quiebra la base del pecíolo (Fig. 4 B y C). Las flores y frutos muy nuevos también pueden ser afectados, los cuales se necrosan parcial o totalmente sin haber aún asomado del cáliz (Fig. 4 D). En frutos un poco más desarrollados, la hembra coloca los huevos debajo de la base de los sépalos, donde las larvas raspan la superficie del fruto joven causando cicatrices necróticas que deforman la base de este, quitándole valor comercial (Fig. 4 E). Las plantas dañadas desde muy jóvenes pueden ser severamente limitadas en su desarrollo y producción (Fig. $4 \mathrm{~F}$ ).

En las dos parcelas de tomate bajo estudio, se dieron situaciones contrastantes en cuanto a infestación por P. longifila. En la primera (junio-octubre) aunque a los 11 días postrasplante, se mantenía bajo porcentaje de infestación, con 14,05+5,36\% (media \pm error estándar de la media) de plantas mostrando ligeros daños en la parte apical, aumentó a $48,77 \pm 11,62 \%$ a los 18 días, para alcanzar el $100 \%$ de infestación con fuertes daños a los 25 días. Como resultado, el desarrollo vegetativo estuvo severamente afectado, con muy escasa ramificación en

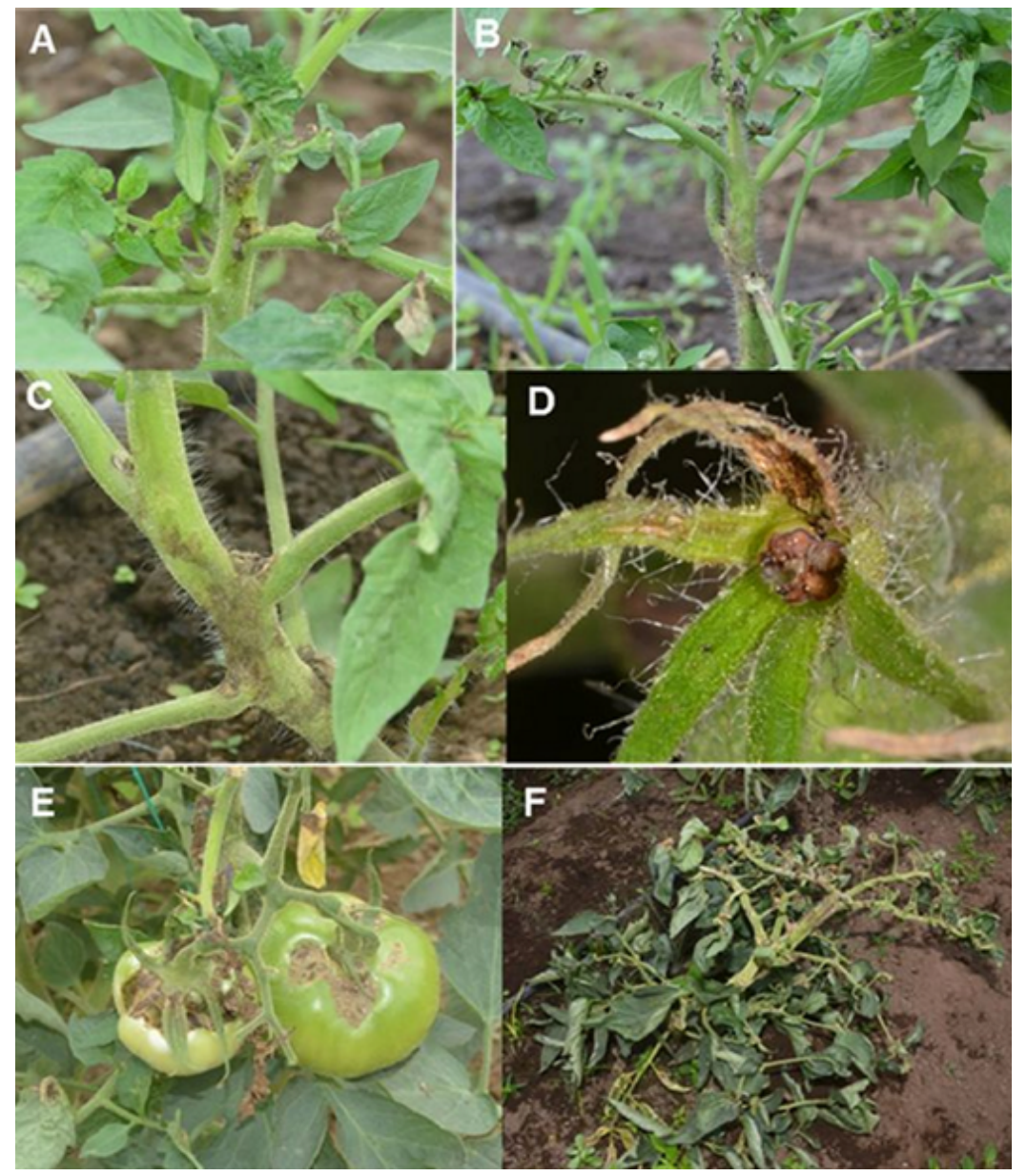

Figura 4. Daños por Prodiplosis longifila en plantas de tomate. A. Daños iniciales en folíolos y brote. B. Hojas con folíolos necrosados y quiebre de pecíolo (flecha indicadora). C. Daño en yemas axilares. D. Daño en fruto muy joven. E. Daño en base de frutos desarrollados. F. Planta con daños severos. 
las plantas y la producción no pasó de un par de decenas de frutos, todos con severos daños por este insecto. La alta infestación y consecuente daño a las plantas de tomate por $P$. longifila, que se hizo aparente entre el cuarto y sexto conteo, de seguro limitó la disponibilidad de sustrato para el insecto, y por ende su desarrollo poblacional, fenómeno este conocido como condicionamiento por daño causado a la planta (Karba y Baldwin 1997; Latimer y Oetting 1999). No obstante, hubo suficiente disponibilidad de material vegetativo para permitir los muestreos por cinco semanas más.

En la segunda parcela de observación (noviembre 2015-enero 2016), a pesar de que se trasplantó en el mismo sitio con poco menos de un mes de intervalo, la infestación por $P$. longifila, se mantuvo muy baja. El porcentaje de plantas infestadas detectado a los 20 días del trasplante fue de $11,23 \pm 1,17$, después de lo cual declinó. Hubo buen desarrollo vegetativo de las plantas (aquellas que no fueron afectadas por Verticillium sp.), con normal ramificación a partir de las yemas axilares y la fructificación no se vio mayormente afectada por este insecto, solo mostrando ligeros daños en muy pocos frutos. Los pesos frescos de muestras tomados en la primera y segunda parcela resultaron $10,78 \pm 0,93 \mathrm{~g}$ y $5,99 \pm 1,14 \mathrm{~g}$, respectivamente. Esa disminución del peso de muestras en la segunda parcela se debió a la dificultad para colectar material vegetativo con daños recientes, debido a la baja infestación. De hecho, durante el quinto muestreo, apenas se lograron colectar dos muestras de las usuales 15.

Esa diferencia puede ser en parte debida al proceso de sucesión ecológica para ese cultivo como comunidad biótica (Price y Walbauer 1994). Tomando en cuenta que en El Misionero no se sembró tomate los dos años previos, al sembrarlo nuevamente, poblaciones residuales de $P$. longifila, sobre otras plantas hospederas en los alrededores, comenzaron la colonización de la nueva parcela del cultivo, posteriormente seguido por sus parasitoides, cuyo efecto se continuó en la segunda parcela. En la zona son muy comunes las plantas de higuerilla, la cual es conocida como hospedera silvestre de $P$. longifila (Díaz 1981; Valarezo et al. 2003; Díaz-Silva 2011).

Identificación de Prodiplosis longifila y sus parasitoides. Adultos hembras y machos de P. longifila obtenidos de las muestras de campo procesadas en el laboratorio, coincidieron con las descripciones de Gagné (1986). En cuanto a parasitoides se separaron ocho morfotipos de Platygastridae y un Chalcidoidea (ambos Hymenoptera) (Fig. 5). Uno de los Platygastridae coincide en su morfología con una especie referida como Synopeas aff. curvicauda Förster, 1896, en Colombia por Hernández (2014) y muy parecido a Synopeas sp. mencionado en Perú (Díaz-Silva 2011); una especie con el gaster de forma muy conspicua (Fig. 5 B). Dos de los otros morfotipos corresponden con hembra y macho de otra especie de Synopeas (Fig. 5 E y H), morfológicamente diferente a las especies encontradas en Colombia (Hernández 2014); la hembra tiene el ovipositor visiblemente sobresaliente (Fig. 5 E). Además, existe una especie de Leptacis Förster, 1856 (Fig. 5 C) y una de Platygaster Latreille, 1809 (Fig. 5 D) (Peter Neerup Buhl, comunicación personal). Los otros tres morfotipos (Fig. 5 A, F y G), corresponderían a machos de $S$. aff. curvicauda; aunque existen dudas con el primero de ellos, por el menor tamaño de los especímenes y color marrón de las patas vs. patas con tibias negruzcas en los otros de mayor tamaño. La especie de Chalcidoidea (Fig. $5 \mathrm{~J}$ ), difiere de lo que Hernández (2014) mencionó como Especie morfo 1.

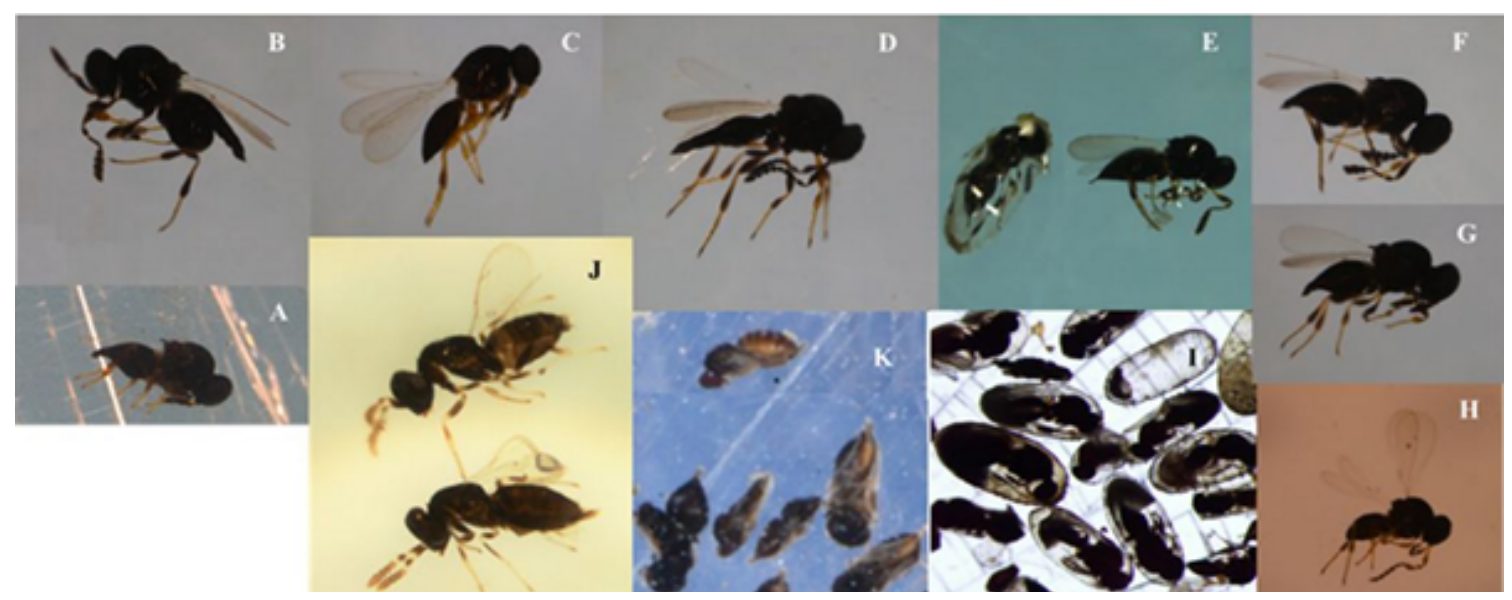

Figura 5. Especies y morfotipos de Hymenoptera parasitoides asociados con Prodiplosis longifila Gagné en tomate. A. Morfotipo 1 (Synopeas. aff. curvicauda ${ }^{\text {}}$ ). B. Morfotipo 2 (S. aff. curvicauda P). C. Morfotipo 3 (Leptacis sp.). D. Morfotipo 4 (Platygaster sp.). E. Morfotipo 5 (Synopeas sp. ㅇ). F. Morfotipo 6 (S. aff. curvicauda ${ }^{\top}$ ). G. Morfotipo 7 (S. aff. curvicauda

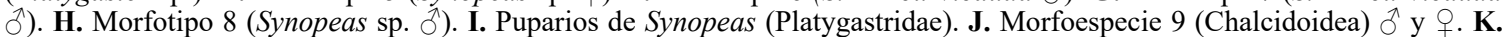
Pupas de morfoespecie 9. Parcelas de tomate en Centro Experimental Misionero, cantón Milagro, provincia de Guayas, Ecuador.

Las nueve especies de parasitoides asociadas con P. longifila, cinco en Colombia (Hernández 2014) junto con las cuatro nuevas (sin repetir a $S$. aff. curvicauda) aquí mencionadas para Ecuador, detectadas durante el reciente período 2012-2016, contrastan con una sola especie hasta entonces conocida en Perú, Synopeas sp. (Díaz-Silva 2011) y mencionada en Ecuador (Valarezo et al. 2003), desde que hubo regis- tros verificados de $P$. longifila en Perú a fines de la década de 1950 (Díaz 1981). Esto denota que durante largo tiempo (> 50 años) no se le prestó suficiente atención a la diversidad de parasitoides, así como a desarrollar métodos que permitieran detectarlos. El contenedor de muestras desarrollado para esta investigación facilitó considerablemente criar esa diversidad de parasitoides, así como conocer aspectos de sus relaciones 
condiciones, en tan corto período de tiempo (junio 2015- febrero 2016) y limitada cobertura geográfica (cantón Milagro, provincia de Guayas, Ecuador).

Las especies de Synopeas encontradas pupan dentro del integumento momificado de la larva de tercer estadío del hospedero, después que esta ha tejido su propio capullo de seda y muere por efecto del parasitismo (doble pupario) (Fig. 5 I). Por el contrario, la pupa del Chalcidoidea queda desnuda dentro del capullo del hospedero, al despojarse la larva del integumento de su hospedero muerto por el parasitismo, antes de pupar (Fig. $5 \mathrm{~K}$ ). Esta diferencia fue detectada al disolver los capullos de P. longifila con hipoclorito de sodio. Obviamente, en ambos casos se trata de endoparasitoides solitarios y la larva hospedera muere en prepupa. El Chalcidoidea alcanzó hasta el 38\% del parasitismo total. Synopeas sp. mencionado en Florida, es parasitoide de huevo-larva de $P$. longifila (Peña et al. 1989). El control biológico de este insecto por Synopeas spp. ha demostrado ser efectivo en huertos de limas en el sur de Florida (Peña et al. 1990).
Comparación de los manejos de muestras. Una de las principales diferencias entre los dos manejos de muestras utilizados, estuvo en el tiempo que toma el procesamiento de estas en el laboratorio, en cuanto a separar y poner en cría los individuos obtenidos del follaje infestado, así como la cantidad de esos individuos y de adultos criados a partir los mismos. Los dos últimos nos permiten estimar las poblaciones del insecto fitófago y la incidencia de sus parasitoides. No hubo apreciables diferencias, en los tiempos de toma de muestras en el campo, aunque resultó más sencillo colocar los brotes y folíolos infestados sobre la rejilla dentro de los contenedores con suelo, que envolverlos en el papel toalla, para colocarlos dentro de la bolsa de polietileno.

Comparando los tiempos de procesamiento de las muestras en laboratorio, aquellas en bolsas de polietileno, envueltas en papel toalla, tomaron $20-30 \mathrm{~min} /$ muestra, dependiendo del número de larvas de $P$. longifila emergidas del follaje. La mortalidad de apreciable número de larvas atrapadas en la humedad sobre las paredes internas de la bolsa, además de

Tabla 1. Individuos obtenidos de muestras, adultos y parasitoides emergidos de sus crías, porcentajes de parasitismo, para Prodiplosis longifila en tomate, mediante dos métodos de muestreo evaluados en lote de observación en el Centro de Investigación El Misionero, cantón Milagro, provincia de Guayas. Ecuador. Período junio-octubre 2015.

\begin{tabular}{|c|c|c|c|c|c|}
\hline Método de muestreo & $\begin{array}{l}\text { Obtenidos de } \\
\text { muestras (1) }\end{array}$ & $\begin{array}{c}\text { Adultos P. } \\
\text { longifila (2) }\end{array}$ & Parasitoides (3) & $\begin{array}{l}\text { Adultos totales } \\
\text { (4) }(=2+3)\end{array}$ & \%Paras. (3/4) \\
\hline Bolsas (A) & $70,8 \pm 1,75$ & $23,5 \pm 1,14$ & $6,1 \pm 0,47$ & $33,8 \pm 1.61$ & $19,8 \pm 1,29$ \\
\hline \multicolumn{6}{|l|}{$N=47$} \\
\hline Tasa $(2,3,4 / 1)$ & & $0,35 \pm 0,04$ & $0,08 \pm 0,01$ & $0,51 \pm 0,06$ & \\
\hline Contenedores (B) & $354,5 \pm 29,42$ & $146,9 \pm 18,42$ & $48,6 \pm 6,77$ & $195,5 \pm 23,34$ & $29,7+4,16$ \\
\hline \multicolumn{6}{|l|}{$\mathbf{N}=\mathbf{5 8}$} \\
\hline Tasa $(2,3,4 / 1)$ & & $0,40 \pm 0,02$ & $0,14 \pm 0,02$ & $0,53 \pm 0,02$ & \\
\hline $\mathbf{B} / \mathbf{A}$ & 5 & 6,2 & 7,7 & 5,8 & 1,5 \\
\hline Contenedores (C) & $238,2+33,62$ & $99,3 \pm 14,92$ & $30,2 \pm 5,90$ & $129,5 \pm 19,88$ & $25,6 \pm 3,60$ \\
\hline \multicolumn{6}{|l|}{$N=154$} \\
\hline Tasa $(2,3,4 / 1)$ & & $0,45+0,03$ & $0,11+0,01$ & $0,57 \pm 0,03$ & \\
\hline C/A & 3,4 & 4,2 & 4,9 & 3,8 & 1,3 \\
\hline
\end{tabular}

Medias \pm error estándar de medias

daños mecánicos al separar individuos pupando en el papel toalla (Fig. 2 C-F), disminuye el rendimiento de la muestra y altera la estimación de la infestación, apartando posibles efectos sobre la estimación del parasitismo. Además, requiere de estereoscopio para las extracciones de puparios del papel toa1la. Cuando se trata de muestras en contenedores con suelo, el cernido no toma más de $5 \mathrm{~min} /$ muestra, independientemente del número de puparios, excepto por su conteo, el cual se facilita considerablemente al colocarlos sobre una hoja de papel blanco, con la cual contrastan por las partículas de suelo que los envuelve, separándolos a simple vista con un pincel fino (Fig. 3 D-F). En esa operación no se causa mortalidad por manipuleo de los individuos.
En la tabla 1 se muestra que los promedios de individuos obtenidos y puestos en cría (1), adultos de $P$. longifila (2), parasitoides (3) y adultos totales (4), emergidos de dichas crías, en los tres primeros muestreos (B/A), resultaron 5,0-7,7 veces mayores en las muestras recolectadas con contenedores con suelo (B), comparado con las recolectadas con bolsas de polietileno (A), mientras que los porcentajes de parasitismo solo fueron 1,5-2,1 veces mayores. Cuando para las mismas comparaciones se tomaron en cuenta todas las muestras colectadas en contenedores (C/A), las diferencias mantuvieron consistencia con las anteriores, aunque resultaron algo menores, $3,4-4,9$ y 1,3-1,8 veces mayores para número de individuos y porcentajes de parasitismo, respectivamente. Esa disminución 
aparentemente se debe a la dinámica de la relación planta-insecto fitófago-parasitoides, afectada por el deterioro de las plantas en la fase final del estudio. Las apreciables diferencias de obtención de individuos entre los dos tipos de muestras, se debe a la mortalidad de larvas atrapadas en la humedad de las bolsas de polietileno. No obstante, las tasas de emergencia de adultos totales con respecto a los individuos obtenidos de las muestras se igualan entre los dos casos, aparentemente debido a similares proporciones de mortalidad durante las crías en ambos manejos de muestras. En el caso de las muestras en contenedores, cuando se disolvieron los capullos con hipoclorito de sodio, se observaron larvas y pupas de $P$. longifila muertas aparentemente por desecación, flotando en el líquido. Dicha mortalidad no se evaluó. Hasta qué punto influyó la sequedad del suelo finamente tamizado que se colocaba en el contenedor para que el insecto pupara, así como las condiciones de humedad dentro de los viales, son factores que deben ser investigados. En el campo, el suelo donde el insecto pupa, suele mantener cierto grado de humedad por los riegos. De ser este el caso, pudiese ser fácil de subsanar manejando la HR en el sistema de crías, lo cual permitiría mejor expresión de las poblaciones y parasitismo, así como reconocer la diversidad de especies involucradas. Obviamente los contenedores lucen más ventajosos, tanto por el rendimiento como por lo sencillo del manejo.

Tabla 2. Correlaciones (coeficientes) entre individuos obtenidos de muestras de tomate vs. adultos totales (Prodiplosis longifila + parasitoides), adultos de $P$. longifila solo y de sus parasitoides criados de esos individuos. Comparación de dos métodos de muestreo

\begin{tabular}{cccc}
\hline $\begin{array}{c}\text { Método de } \\
\text { muestreo }\end{array}$ & Adultos totales & $\begin{array}{c}\text { Adultos de } \\
\text { P. longifila }\end{array}$ & Parasitoides \\
\hline & & \\
\hline $\begin{array}{c}\text { Bolsas de } \\
\text { polietileno (A) }\end{array}$ & $0,619^{* *}$ & $0,459^{* *}$ & $0,534^{* *}$ \\
\hline $\mathrm{N}=47$ & & \\
\hline \multicolumn{3}{c}{} \\
\hline Contenedor $(\mathbf{B})$ & $0,901^{* *}$ & $0,882^{* *}$ & $0,639^{* *}$ \\
\hline $\mathrm{N}=154$ & & \\
\hline & & \\
\hline $\mathbf{B} / \mathbf{A}$ & 1,46 & 1,92 \\
\hline
\end{tabular}

$* * \mathrm{P}<0,01$

\section{Bolsas de polietileno con papel Contenedores con suelo}

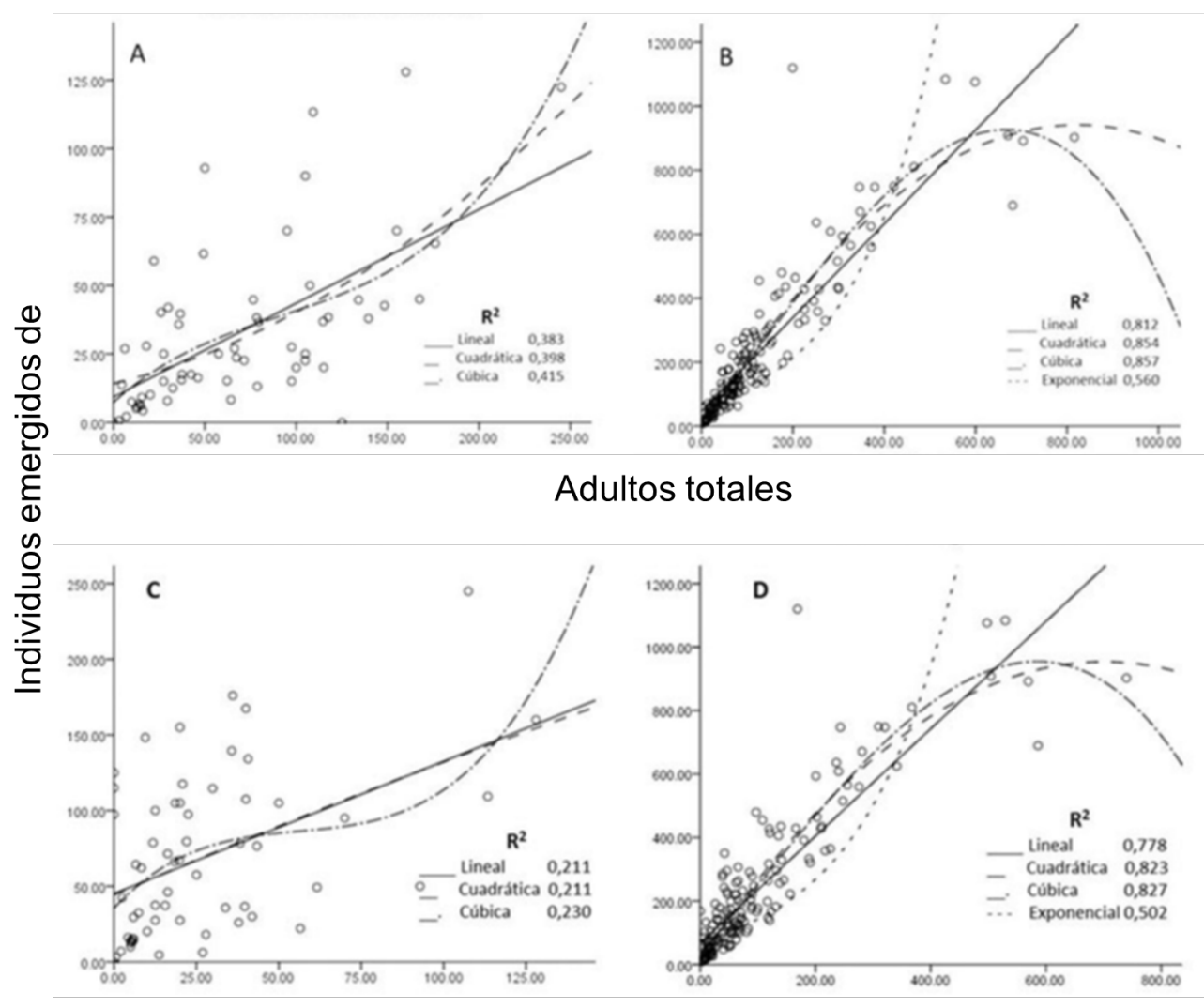

Adultos Prodiplosis

Figura 6. Comparación de muestreos de Prodiplosis longifila en tomate, utilizando bolsas de polietileno y papel toalla (A y C) vs. contenedores de muestras con suelo (B y D), mediante regresiones simples de adultos criados a partir de individuos obtenidos de las muestras vs. adultos totales ( $P$. longifila + parasitoides) (A y B) y adultos de $P$. longifila solo (C y D). Muestreos realizados en lotes de observación en el Centro de Investigación El Misionero, cantón Milagro, provincia de Guayas. Ecuador. Período junio-octubre 2015. Para todos los valores de R2 P $<0,01$. 
Los coeficientes de correlación entre número de individuos obtenidos de las muestras y los adultos emergidos de sus crías resultaron 1,2-1,9 veces mayores para muestras colectadas en contenedores, comparadas con las colectadas en bolsa (Tabla 2). Esto denota mayor exactitud de este método. En el caso de parasitoides solos, las diferencias entre los tipos de muestreo resultaron menores. Ello podría deberse a que el sistema de muestreo afecta el número de individuos obtenidos de la muestra que llegan a completar su ciclo hasta adulto, pero no la proporción de individuos parasitados, a menos que el parasitismo sea susceptible al manejo diferencial de las muestras. Las correlaciones entre los números de cada taxón de parasitoide y el parasitismo total indican que Synopeas spp. son algo más determinantes de dicho parasitismo total $(\mathrm{r}=$ $0,7949, p<0,05)$ comparado con el Chalcidoidea $(r=0,5939$, $\mathrm{p}<0,05)$.

Al comparar mediante ecuaciones de regresión los adultos totales y de $P$. longifila solo, obtenidos de los individuos recuperados de las muestras dentro de bolsas de polietileno, con las colocadas dentro de contenedores, observamos que las primeras (Fig. $6 \mathrm{~A} \mathrm{y} \mathrm{C)} \mathrm{presentan} \mathrm{puntos} \mathrm{muy} \mathrm{dispersos}$ produciendo bajos valores de $\mathrm{R}^{2}$ para todas las ecuaciones (amplitud A: 0,383-0,415; C: 0,211-0,230), lo cual indica débil asociación entre individuos obtenidos de esas muestras y los que sobreviven hasta llegar a adultos. Por el contrario, cuando se trata de muestras en contenedores (Fig. 6 B y D) las relaciones entre esos valores son mucho más estrechas, produciendo puntos más agrupados alrededor de las líneas es- timadas por cada modelo, lo que produjo altos valores de $\mathrm{R}^{2}$ (amplitud B: 0,812-0,857; D: 0,778-0,817). Evidentemente, este es un método más confiable.

Fluctuación poblacional de $P$. longifila y sus parasitoides. Las gráficas de la Figura 7 A y B, muestran las fluctuaciones poblacionales de P. longifila y porcentajes de parasitismo, en la primera y segunda parcela respectivamente. En la Figura 7 A se puede observar cómo la población de P. longifila en la primera parcela de tomate (julio-octubre 2015) sufrió un acentuado incremento entre la cuarta y séptima semana, período durante el cual también se acentuó la diferencia entre número de individuos obtenidos de las muestras y adultos de $P$. longifila criados de esos individuos en el laboratorio. Aunque el parasitismo aumentó durante ese periodo, dicho aumento no compensa esas diferencias. La mortalidad por aparente desecación observada durante el procesamiento de las muestras en el laboratorio podría ser responsable en parte de esa diferencia. La disminución de la población de $P$. longifila, después del pico poblacional, de seguro estuvo al menos en parte influenciado por el visible deterioro de las plantas consecuencia del daño por el insecto.

En la segunda parcela de tomate (Fig. 7 B), con niveles poblacionales parecidos al inicio de la primera, la mayoría de los individuos que se obtuvieron de las muestras se convirtieron en adultos. En todo caso, la sola evaluación de los puparios es un buen índice de la infestación, solo que los números de adultos del insecto fitófago y sus parasitoides producidos de

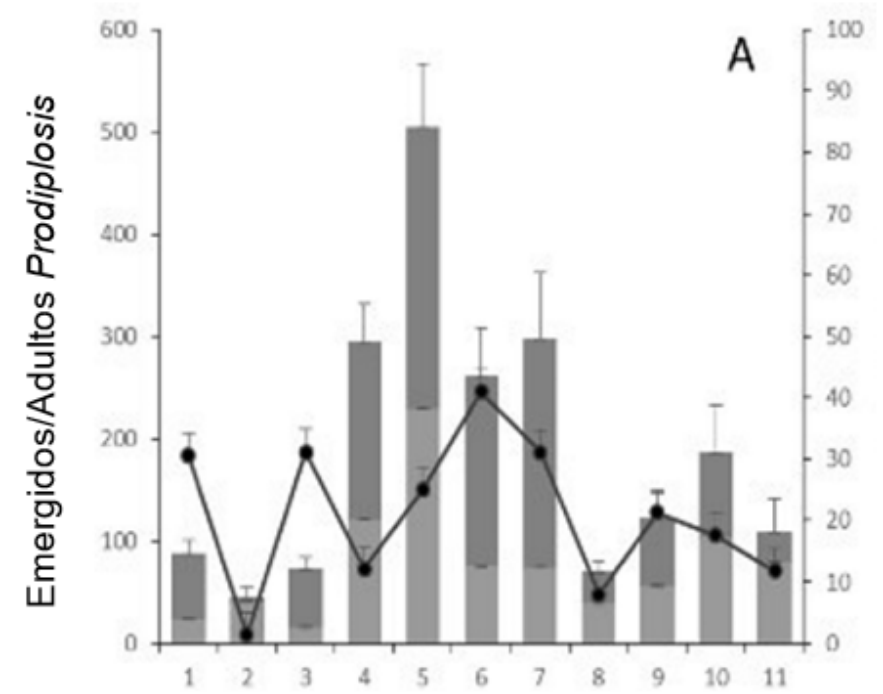

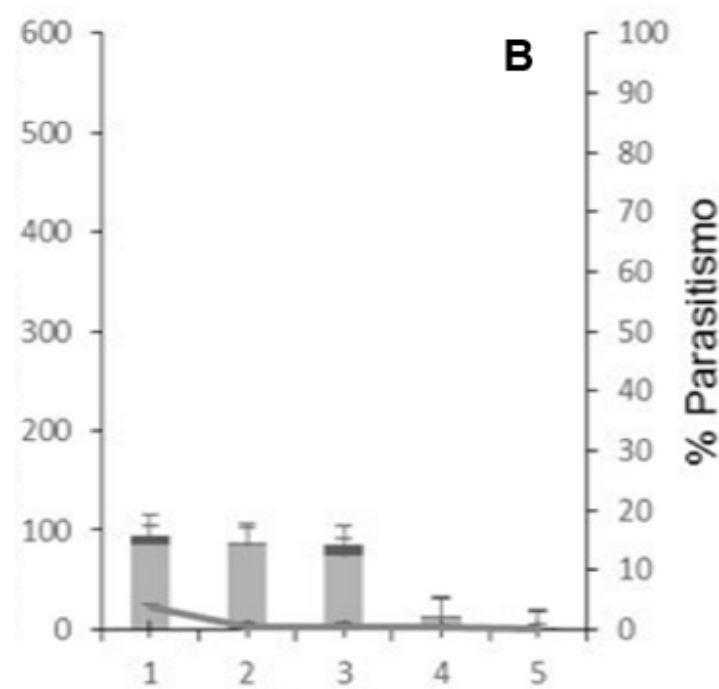

Semanas

Figura 7. Fluctuación del número de individuos de Prodiplosis longifila obtenidos de muestras de plantas de tomate (barras completas), adultos criados de los esos individuos (parte baja de las barras en gris claro) y porcentaje de parasitismo. Lotes de observación en el Centro Experimental El Misionero, Milagro, Guayas. Períodos A. Junio-octubre 2015 y B. Noviembre-enero 2016. 
esos individuos obtenidos estarían subestimados en proporción a esas diferencias entre obtenidos y adultos producidos; cosa que debe ser dilucidada como ya se mencionó, mediante evaluación de condiciones óptimas de crías de individuos dentro de sus puparios en el laboratorio.

\section{Conclusiones}

La manera como surgieron los problemas fitosanitarios causados por $P$. longifila alrededor de 50 años después de haber sido detectada (1930-1979), sugieren causalidad por impacto de manejos agronómicos, especialmente en lo que refiere al uso de plaguicidas organosintéticos, sin descartar posible efecto adicional de diversificación genética de las poblaciones a lo largo de esa amplia distribución geográfica, sus variantes climáticas y manejos agronómicos.

La diversidad de especies de parasitoides de $P$. longifila a lo largo de su distribución geográfica en Suramérica, aquí documentada, sugiere que este insecto estaba presente mucho antes de convertirse en problema. Esto denota que inicialmente no se prestó suficiente atención al control biológico natural, tratando de resolver el problema utilizando las tecnologías químicas que muy probablemente causaron los desbalances que lo originaron. Esto explicaría por qué el control biológico natural "aquí no ha funcionado", en contraposición de lo que ocurre en Florida (EE.UU.). Ese enfoque unilateral de manejo de "plagas" es lo que ha llevado al incremento actual de insecticidas utilizado contra $P$. longifila, lo cual es absolutamente insostenible.

El manejo de muestras usando contenedor con suelo para estudiar poblaciones de $P$. longifila y diversidad de parasitoides, resultó más eficiente, obteniéndose adultos de buena calidad para fines taxonómicos. También es potencialmente utilizable para otros insectos fitófagos pequeños que pupen en el suelo, así como aquellos parasitoides que emerjan de esos puparios. No obstante, es necesario prestarle atención a la mortalidad durante la puesta en cría de los puparios.

El desarrollo y afinamiento de metodologías de muestreo para aproximar el mejor conocimiento de este problema fitosanitario, a nivel poblacional, permitirá avanzar en la racionalización de su manejo desde los puntos de vista ecológico y socioeconómico, disminuyendo el fuerte impacto ambiental y en salud humana que actualmente representa.

\section{Agradecimientos}

Esta investigación se condujo dentro del Proyecto Prometeo de la SENESCYT, en la Universidad Agraria del Ecuador (UAE), Especial agradecimiento a la Revista Colombiana de Entomologia por asumir solidariamente el costo de publicación, al no haber sido cubierto por la UAE. Otto Caicedo, Deiby Duarte, Bryan Martínez, Génesis Valverde, María Díaz y Paul Guamán, estudiantes de Ingeniería Agronómica en la sede Milagro de la UAE, prestaron valiosa colaboración en los trabajos de campo. Así mismo Sylvana Larrea y Ronald Estrella, estudiantes de la UAE Guayaquil, colaboraron con los trabajos en el laboratorio. Dorys T. Chirinos nos orientó a estudiar ese problema sugiriendo revisar su historia, además junto a Rosana Castro ayudaron con la coordinación de estudiantes colaboradores de trabajos en campo y laboratorio. Jaime Pineda facilitó el uso del equipo óptico de alta capacidad. Yoansi García y Ángel Gómez colaboraron con los análisis estadísticos. Juan A. Vergara López, colaboró en la edición de tablas y figuras. Peter Neerup Buhl identificó Platygastridae. José Hernández-Rosas y Ângel Ramírez-Isea revisaron el manuscrito aportando valiosas sugerencias, al igual que las observaciones críticas al manuscrito hechas por los árbitros. A todos ellos, nuestro mayor agradecimiento.

\section{Literatura citada}

CEDANO, C.; CUBAS, P. 2012. Bauveria bassiana (Bals) y Metarhizium anisoliae (Metsch) Sorokin para el control de pupas de Prodiplosis longifila Gagné en el cultivo de espárrago. Scientia Agropecuaria 3 (1): 29-34. https://doi.org/10.17268/sci.agropecu.2012.01.04

CHIRINOS, D. T.; GERAUD-POUEY, F. 1996. Efectos de algunos insecticidas sobre la entomofauna del tomate, en el noroeste del estado Zulia, Venezuela. Interciencia 21 (1): 31-36.

CHIRINOS, D. T.; GERAUD-POEUY, F. 2011. El manejo de plagas agrícolas en Venezuela. Reflexiones y análisis sobre algunos casos. Interciencia 36 (3): 192-199.

CHIRINOS, D. T.; DIAZ, A.; GERAUD-POUEY, F. 2014. El control biológico ejercido por parasitoides sobre el minador de la hoja del cebollín, Liriomyza trifolii (Burgess). Entomotrópica 29 (3): 129-138. https://www.interciencia.net/wp-content/ uploads/2018/01/192-CHIRINOS-8.pdf

CHIRINOS, D. T., CASTRO, R., CUN, J., CASTRO, J., PENARRIETA, S., SOLIS, L., \& GERAUD, F. 2020. Los insecticidas y el control de plagas agrícolas: la magnitud de su uso en cultivos de algunas provincias de Ecuador. Ciencia y Tecnología Agropecuaria, 21(1), e1276. https://doi.org/10.21930/rcta. vol21_num1_art:1276

DHILEEPAN, K.; NESER, S.; RUMIZ, D.; RAMAN, A.; SHARMA, A. 2017. Host associations of gall-inducing Prodiplosis longifila (Diptera: Cecidomyiidae) from Bolivia: implications for its use as a Biological Control agent for Jatropha gossypiifolia (Euphorbiaceae). Florida Entomologist 100 (4): 777-786. https://doi.org/10.1653/024.100.0402

DÍAZ, W. 1981. Prodiplosis sp. (Diptera: Cecidomyiidae) plaga de la alfalfa y otros cultivos. Revista Peruana de Entomología 24 (1): 95-97.

DÍAZ-SILVA, F. F. 2011. Aspectos agroecológicos para el Manejo Integrado de Prodiplosis longifila Gagné en la Irrigación Chavimochic, 1994-2010". Monografía Científica. Facultad de Ciencias Biológicas. Universidad Nacional de Trujillo. 93 p. Disponible en: http://www.academia.edu/15424714/Aspectos_Agroecol\%C3\%B3gicos_para_el_Manejo_Integrado_de_Prodiplosis_longifila_Gagn $\% \mathrm{C} 3 \% \overline{\mathrm{A}} 9$-en_la_Irrigaci\% $\% \overline{\mathrm{C}} \% \overline{\mathrm{B} 3} \mathrm{n}$ _Chavimochic [fechà de revisión: 13 abril 2016].

DOUTT, R. L.; SMITH, R. F. 1971. The pesticide syndrome- Diagnosis and suggested prophylaxis. Cap.1 En: Huffaker, C.B. (Ed.). Biological Control. Plenum. New York. 511 p.

ESPINOSA, L. L.; TORO, N. 2015. Influencia de la cordillera Central colombiana en la estructura poblacional de Prodiplosis longifila (Diptera: Cecidomyiidae). 20 p. http://bibliotecadigital. univalle.edu.co/bitstream/10893/7924/1/CB-0492874.pdf [fecha de revisión: 20 mayo 2016].

GAGNÉ, R. J. 1986. Revision of Prodiplosis (Diptera: Cecidomyiidae) with descriptions of three new species. Annals of the Entomological Society of America 79: 235-245. https://doi. org/10.1093/aesa/79.1.235

GERAUD-POUEY, F.; CHIRINOS, D. T.; RIVERO, G. 1995. Artrópodos asociados con el cultivo del tomate (Lycopersicon esculentum Mill.) en Venezuela. Boletín de Entomología Venezolana 10 (1): 31-49.

GERAUD-POUEY, F.; CHIRINOS, D. T.; GALINDO-CASTRO, I.; FRANCO, M. A.; SANTANA, M. A.; GILLIS, A.; ROMAY, G. 2015. Occurrence of six begomoviruses infecting tomato fields in Venezuela and genetic characterization of Potato Yellow Mosaic Virus isolates. Journal of Phytopathology 164, 697-703. http://dx.doi.org/10.1111/jph.12445. 
GROUSSET, F.; SUFFERT, M.; PETTER, F. 2015. Study on pest risk associated with the import of tomato fruit. OEPP/EPPO Bulletin 45 (1): 153-156. https://doi.org/10.1111/epp.12180

HERNÁNDEZ, L. M. 2014. Caracterización del daño y distribución geográfica de Cecidomyiidae (Diptera) y sus parasitoides asociados a solanáceas y limón Tahití en Colombia. Tesis Magister en Ciencias Agrarias. Universidad Nacional de Colombia. Palmira, Colombia. 94 p. Disponible en: http://www.bdigital.unal. edu.co/17947/1/7211002.2014.pdf [fecha de revisión: 15 marzo 2016].

HERNÁNDEZ, L. M.; GUZMAN, Y. C.; MARTÍNEZ-ARIAS, A.; MANZANO, M. R.; SERVARAJ, J. J. 2015. The bud midge Prodiplosis longifila: damage characteristics, potential distribution and presence on a new crop host in Colombia. Springer Plus 4: 205. https://doi.org/10.1186/s40064-015-0987-6

HERRERA, J. M. 2010. Primera experiencia a nivel mundial de Manejo Integrado de Plagas: el caso del algodonero en el Perú. Revista Peruana de Entomología 46 (1): 1-8.

IBM Corp. 2011. IBM SPSS Statistics for Windows, versión 20.0. Armonk, NY.

KARBA, R.; BALDWIN, I. T. 1997. Induced responses to herbivory. The University of Chicago Press. Chicago y Londres. 319 p.

LAMAS, G. 1982. Bibliografía de Johannes E. Wille (1892-1959). Revista Peruana de Entomología 25 (1): 87-94.

LATIMER, J. G.; OETTING, R. D. 1999. Conditioning treatments affect insect and mite populations on bedding plants in the greenhouse. HortScience 34 (2): 235-238. https://doi.org/10.21273/ HORTSCI.34.2.235

MENA, Y. M.; MESA, N. C.; ESTRADA, E. I.; GARCIA, Y. 2014. Evaluación de la resistencia a Prodiplosis longifila Gagné (Diptera: Cecidomyiidae) en genotipos de tomate cultivados y silvestres. Acta Agronómica (Colombia) 63 (2): 175-180. https://doi. org/10.15446/acag.v63n2.30210

OATMAN, E. R.; KENNEDY, G. G. 1976. Methomyl induced outbreak of Liriomyza sativae on tomato. Journal of Economic Entomology 69 (3): 667-668. https://doi.org/10.1093/jee/69.5.667

ORTEGA, E.; URETA, C.; MAYANGA, A.; MOREY, G.; GRATEROL, L.; FYER, P.; MIALHE, E. 2014. Identificación molecular de la mosquilla del brote Prodiplosis sp. Gagné en los cultivos de Asparagus officinalis L. por ampliación parcial del gen citocromo oxidasa I. Idesia (Chile) 32 (4): 29-41. https://doi. org/10.4067/S0718-34292014000400005

PEÑA, J. E.; BARANOWSKI, R. M.; McMILLAN, R. T., Jr. 1987. Prodiplosis longifila (Diptera: Cecidomyiidae) a new pest of citrus in Florida. Florida Entomologist 70 (4): 527-529. https://doi. org/10.2307/3494798

PEÑA, J. E.; GAGNÉ, R. J; DUNCAN, R. 1989. Biology and characterization of Prodiplosis longifila (Diptera: Cecidomyiidae) on lime in Florida. Florida Entomologist 72 (3): 445-450. https:// doi.org/10.2307/3495182

PEÑA, J. E.; DUNCAN, R.; TORRES, V. 1990. Control of the citrus midge Prodiplosis longifila Gagné in southern Florida limes. Proceedings of the Interamerican Society for Tropical Horticulture. 34: 159-161.

PEÑA, J. E.; DUNCAN, R. 1992. Sampling Methods for Prodiplosis longifila (Diptera: Cecidomyiidae) in Limes. Environmental Entomology 21 (5): 996-1001. https://doi.org/10.1093/ee/21.5.996

PERALTA, I. E.; SPOONER, D.M. 2000. Classification of wild tomatoes: a review. Kurtziana (Argentina) 28: 45-54.

PRICE, P.; WALBAUER, G. 1994. Ecological Aspects of Pest Management. Cap. 2 En: Metcalf, R. L.; Luckmann, W. H. (Eds.). Introduction to Insect Pest Management. Wiley. New York. 650 p.

RAINWATER, C. F. 1934. Insects and a mite of potential economic importance found on wild cotton in Florida. Journal of Economic Entomology 27: 756-761. https://doi.org/10.1093/jee/27.4.756

ROMAY, G.; GERAUD-POUEY, F.; CHIRINOS, D. T.; DEMEY, J. R. 2016. Bemisia tabaci (Gennadius) (Hemiptera: Aleyrodidae): Historia, situación actual y su rol como vector de enfermedades virales de plantas en Venezuela. Entomotrópica 31: 276-293.
VALAREZO, O.; CAÑARTE, E.; NAVARRETE, B.; ARIAS, M. 2003. Prodiplosis longifila (Diptera: Cecidomyiidae) principal plaga del tomate en Ecuador. Instituto Nacional Autónomo de Investigaciones Agropecuarias. Estación Experimental de Portoviejo. (Proyecto IG CV 028). 82 p.

\section{Origen y financiación}

Esta publicación, es producto de lo que por insuficiente financiamiento, se pudo acometer del proyecto originalmente sometido por el autor principal y aprobado por la SENESCYT de la República de Ecuador, para su contratación dentro del Programa Prometeo (junio 2015-junio 2016). A pesar de esas limitaciones, los resultados abren nuevas perspectivas para el manejo de uno de los problemas entomológicos más relevantes en la agricultura de la zona Andina. Su tardía publicación se logró gracias a la Revista Colombiana de Entomología, la cual asumió esos costos, responsabilidad inexplicablemente no honrada por la Universidad Agraria del Ecuador, institución de asignación del Investigador.

\section{Contribuciones de los autores}

FGP: Investigador proponente y responsable del proyecto, coordinación y conducción de investigación en campo, laboratorio y revisión bibliográfica, documentación fotográfica, procesamiento de datos, redacción del manuscrito, revisión y edición.

AG: Responsable agronómico de las parcelas en campo. Administración y suministro de recursos, revisión y validación de resultados. Edición.

NC: Procesamiento de muestras en laboratorio, ordenamiento y procesamiento de datos, validación de resultados. Revisión del manuscrito y edición.

JEGCH: Propagación de plantas y establecimiento de parcelas en campo, evaluación de daños, muestreos en campo y procesamiento en laboratorio. Documentación fotográfica, revisión y edición.

\section{Conflictos de interés}

Los autores declaramos no tener conflictos de intereses. 